\title{
Identification of the active substance and mechanisms of CFF-1 for treatment of prostate cancer based on network pharmacology, virtual screening, and molecular dynamics simulation
}

\section{zao dai}

Nanjing Normal University School of Life Sciences https://orcid.org/0000-0002-1286-3685

Ping Liu ( $\square$ liuping0805@njnu.edu.cn )

Nanjing Normal University School of Life Sciences https://orcid.org/0000-0001-5366-4618

\section{Research}

Keywords: CFF-1, Prostate cancer, Network pharmacology, Virtual screening, Molecular dynamics

Posted Date: April 8th, 2021

DOl: https://doi.org/10.21203/rs.3.rs-401623/v1

License: (c) (1) This work is licensed under a Creative Commons Attribution 4.0 International License. Read Full License 


\section{Abstract \\ Background}

CFF-1 is an effective treatment option for prostate cancer patients, but there is a lack of network pharmacology research on its molecular mechanism.

\section{Methods}

Based on bioavailability and drug-likeness, the main active ingredients of CFF-1 were obtained through the Traditional Chinese Medicine System Pharmacology (TCMSP) database. The GeneCard, OMIM, PharmGKB, Therapeutic Targets database, and DrugBank were used to construct prostate cancer target gene sets. Construct an ingredients-target network and a protein-protein interaction network respectively, and GO and KEGG enrichment analysis were performed. After merging the two networks, important target genes were obtained in the merged network, and virtual screening of CFF-1 active ingredients was performed on these important target genes. Then the dynamic process of the binding of two small drug molecules to the target protein was simulated by molecular dynamics.

\section{Results}

112 active ingredients of CFF- 1 and 206 prostate cancer target genes were identified. The results of GO and KEGG enrichment analysis showed that the target genes regulated by CFF- 1 were involved in prostate cancer-related signaling pathways. Two important target genes were obtained in the merge network, as well as the small molecules with the best binding among the active ingredients of CFF-1 were obtained through a virtual screening tool developed by us. Finally, simulate the dynamic process of two small molecules binding to the target protein.

\section{Conclusion}

In conclusion, this study predicted the potential molecular mechanism of CFF-1 in the treatment of prostate cancer through network pharmacology and found important target genes and their active ingredients in CFF-1. This provided a direction for future research on CFF-1 and promoted the reasonable application of CFF-1 in the clinical treatment of prostate cancer.

\section{Introduction}

Prostate cancer is the second most deadly cancer in males [1]. At present, the main treatment methods for prostate cancer are chemotherapy, radiotherapy, and some combination therapies [2-4]. Because the tumor has the characteristics of metastasis and gene mutation, prostate cancer will still develop and 
metastasize after treatment $[5,6]$. Therefore, new therapy methods are still needed to be developed for treating prostate cancer.

Over-activation of ESR1 and MAPK14 has been reported in prostate cancer $[7,8]$. In the tumor, ESR1 and BARX2 can coordinately regulate cell growth and invasion [9]. Activated MAPK14 can promote IL-1 induced cell proliferation and reduce TNF-induced apoptosis $[10,11]$. The estrogen signaling pathway and MAPK signaling pathway have been reported to be necessary for the development of tumors $[12,13]$.

Traditional Chinese Medicine (TCM) has a long history and unique characteristics. Many researchers have found that TCM can suppress diseases (including cancer) by improving the body's immunity [14]. Also, TCM has little cytotoxicity and side effects during the treatment process [15] and has systemic effects that Western medicine does not have [16]. Although TCM has these unique advantages, the molecular mechanism of its treatment of cancer is still unclear. CFF-1 was a kind of Compound TCM, which was created by famous TCM doctor Fusong Xu of Jiangsu Province Hospital of Traditional Chinese Medicine. Studies have shown that CFF-1 can inhibit the growth of prostate cancer cells and promote cancer cell apoptosis through PI3K-AKT and EGFR-related signaling pathways $[17,18]$. Based on the network relationship of disease, genes, and drugs, network pharmacology is widely used to systematically and comprehensively research the network of drugs in treating diseases and to explain the overall impact of drugs on the body. With the development of bioinformatics, it also provides the possibility for TCM network pharmacology research $[19,20]$. Therefore, it is important to systematically research the molecular mechanism of CFF-1 in the treatment of prostate cancer through bioinformatics.

In this study, the molecular mechanism of CFF-1 inhibition of prostate cancer was researched based on network pharmacology. The Results demonstrated CFF-1 mainly inhibited prostate cancer by inhibiting ESR1 of the estrogen signaling pathway and MAPK14 of the MAPK signaling pathway. By using the virtual screening tool developed by ourselves, the small molecules that best bind to ESR1 and MAPK14 among the TCM components were evaluated. Finally, the dynamic process of the binding of small drug molecules to the target protein was simulated by molecular dynamics. Our results provided new ideas and directions for the clinical and experimental research of CFF-1 in inhibiting prostate cancer in the future.

\section{Materials And Methods}

\section{Compounds and target gene screening of CFF-1}

Based on Fusong Xu's treatment idea, Astragalus (Hedysarum Multijugum Maxim), Rehmannia (Rehmanniae Radix Praeparata), Polygonatum (Polygonati Rhizoma), Curcumae Rhizoma, Turmeric (Curcumaelongae Rhizoma), Polyporus (Polyporus Umbellatus (Pers) Fr), and Licorice were selected for the subsequent analysis. According to TCMSP database [21], the chemical composition of CFF-1 was obtained, and the screening condition were oral bioavailability $(\mathrm{OB})>30 \%$ and drug-likelihood $(\mathrm{DL})>0.18$ [20]. Then potential targets of CFF-1 were collected in the TCMSP database and annotated with the UniProt database (https://www.uniprot.org/). 
Based on multiple databases, including GeneCard (https://www.genecards.org/), OMIM (https://www.omim.org/), PharmGkb (https://www.pharmgkb.org/), Therapeutic Targets database (TTD) (http://bidd.nus.edu.sg/group/cjttd/) and DrugBank (https://www.drugbank.ca/) databases, total genes related to prostate cancer were obtained; and then these total genes were combined with total target genes of CFF-1 active ingredients (screened by TCMSP database) to obtain the target genes of CFF-1 active ingredients in prostate cancer.

\section{GO and KEGG enrichment analysis}

The function and pathway enrichment analysis of the final intersection genes of CFF-1 was performed by clusterProfiler [22] and the cancer pathways analyzed from the KEGG database were presented (https://www.genome.jp/kegg/).

\section{Construction of protein-protein interaction (PPI) network and core gene screening}

Constructed PPI network with STRING database [23] of CFF-1 target genes and displayed them with Cytoscape [24]. According to six features defined by CytoNCA [25] (degree centrality (DC), betweenness centrality (BC), closeness centrality (CC), eigenvector centrality (EC), network centrality (NC), and local average connectivity (LAC)), the importance of PPI network nodes was calculated and core genes PPI network was built.

\section{Construction of merge network}

According to the relationship between active ingredients and their target genes screened out from the TCMSP database, an ingredient-target network was constructed with Cytoscape. Combined the PPI network of core genes with the ingredient-target network of Cytoscape, the merged network of core targetgenes was constructed.

\section{Virtual screening.}

According to the merged network, the three-dimensional structure of the proteins from the Research Collaboratory for Structural Bioinformatics Protein Data Bank (RSCB PDB) database

(https://www.rcsb.org/) was obtained. Based on the regulatory relationship between target genes and chemical ingredients in the merged network, the structures of chemical ingredients were obtained from the PubChem database (https://pubchem.ncbi.nlm.nih.gov/) of National Center for Biotechnology Information (NCBI). To obtain the results of the smallest binding affinities between the target proteins and the chemical ingredients in CFF-1, we developed a virtual screening tool named Do_virtual_screening (https://github.com/daizao/Do_virtual_screening) based on AutoDock Tools [26] and AutoDock Vina [27]. After analysis using our screening tool, the minimum binding affinities of each target protein were obtained. The three-dimensional interactions between ligand and target protein were presented by Pymol 
(https://github.com/schrodinger/pymol-open-source) and two-dimensional putative docking pose of ligand in the binding pocket was exhibited by LigPlot [28].

\section{Molecular dynamics simulation}

Perform molecular dynamics simulations with Gromacs [29] (2018.3 version) on the two core proteins and the top-ranked ligand. And to make the simulation of protein structure and ligand reach a balanced state, the two protein and ligand complexes were simulated for $100 \mathrm{~ns}$ and $150 \mathrm{~ns}$, respectively.

\section{Results}

\section{Screening of target genes of CFF-1 active ingredients in prostate cancer}

The flowchart of this study was shown in Fig. 1. According to the screening results of the TCMSP database, CFF-1 contained 112 active ingredients, and it's found that licorice had the most target genes (Table S1). In table S1, the same target genes could be regulated by different small ingredients from different Chinese medicine, hence the regulation of CFF-1 on prostate cancer was diverse. According to five databases (GeneCards, OMIM, PharmGkb, TTD, and DrugBank), the prostate cancer target genes were obtained (Fig. S1a). After crossing the target genes of the above analysis methods, 206 target genes regulated by CFF-1 were obtained in prostate cancer (Fig. S1b and Table S2).

\section{Biofunctions and pathways of target genes of CFF-1 active ingredients in prostate cancer}

In this study, GO and KEGG enrichment analysis was employed to figure out the functions and pathways of the target genes of CFF-1 active ingredients in prostate cancer. According to the GO enrichment analysis of 206 genes, it was found that functions of these target genes in response to metal ion, membrane raft, and nuclear receptor activity were the most significant in BP (Biological Process), CC (Cellular Component), and MF (Molecular Function), respectively (Fig. 2a). In the results of KEGG enrichment analysis, prostate cancer signaling pathways including IL-17 signaling pathway, EGFR tyrosine kinase inhibitor resistance, and PI3K-Akt signaling pathway, etc. were obtained (Fig. 2b). Besides, other cancer-related pathways, such as colorectal cancer, bladder cancer, small cell lung cancer, hepatocellular carcinoma, and chronic myeloid leukemia (Fig. 2b), were also significantly enriched. Therefore, these target genes (marked in red) played important roles in cancer pathways (including estrogen signaling pathway and MAPK signaling pathway) (Fig. 2c).

\section{Construction of the merge network of core PPI and drug active ingredients}

Based on the screening results of the active ingredient and 206 target genes in the TCMSP database, we constructed a network of active ingredients and target genes (Fig. 3). In this network, many target genes, such as PTGS2, ESR1, MAPK14, AR, etc. could be regulated by a variety of active ingredients of CFF-1. According to the STRING database, the PPI network of 206 target genes was obtained (Fig. 4a). The core 
PPI network was obtained from the PPI network of 206 target genes by analyzing the relationship between nodes in the PPI network of 206 target genes (Fig. 4b). In the core PPI network, the genes, such as TP53, AKT1, MAPK family genes, ESR1, etc. had complex regulatory relationships, indicating that inhibiting these core genes might affect the entire PPI network in prostate cancer. After conjoint analysis of the core PPI network and the network of active ingredients and target genes, the merge network was rebuilt (Fig. 5). In the merge network, ESR1 and MAPK14 were repeatedly regulated by CFF-1 active ingredients, indicating that these two genes played major roles in the process of CFF- 1 inhibiting prostate cancer.

\section{Potential ligands targeting ESR1 and MAPK14 were obtained by virtual screening}

According to the results of Do_virtual_screening (virtual screening tool developed by us, https://github.com/daizao/Do_virtual_screening), we found that Glabrene and (2S)-2-[4-hydroxy-3-(3methylbut-2-enyl)phenyl]-8-8-dimethyl-2-3-dihydropyrano[2-3-f]chromen-4-one had the smallest binding affinities for ESR1 and MAPK14 among 46 ligands (Table S3). The docking result of Glabrene and ESR1 was displayed in 2D and 3D, hydrogen bond interaction between Arg and ligand was found (Fig. 6a). Docking result of 2S)-2-[4-hydroxy-3-(3-methylbut-2-enyl)phenyl]-8-8-dimethyl-2-3-dihydropyrano[2-3f]chromen-4-one and MAPK14 was displayed in 2D and 3D, the hydrophobic interaction played a major role (Fig. 6b).

\section{Molecular dynamics simulation of the binding of ESR1 and MAPK14 to ligands}

Based on the results of the two ligands and protein complexes, molecular dynamics simulations were performed. The RMSD (root-mean-square deviation) of the carbon a skeleton represents the stability of the three-dimensional structure model of the protein-ligand complex during molecular dynamics simulation. After 100ns molecular dynamics simulation of ESR1 and glycyrrhizin, the RMSD value balanced at $0.31 \mathrm{~nm}$ (Fig. 7a). The short-range Coulomb force and the short-range Lanna-Jones potential were $-85(\mathrm{~kJ} / \mathrm{mol})$ and $-182(\mathrm{~kJ} / \mathrm{mol})$ (Fig. 8a) at 100ns, respectively. Hence the complex of ESR1 and ligand was dominated by electrostatic force and Van der Waals force. After 150ns molecular dynamics simulation of MPAK14 and (2S)-2-[4-hydroxy-3-(3-methylbut-2-enyl)phenyl]-8-8-dimethyl-2-3dihydropyrano[2-3-f]chromen-4-one, the RMSD value balanced at $0.53 \mathrm{~nm}$ (Fig. 7b). The short-range Coulomb force and the short-range Lanna-Jones potential were - $6(\mathrm{~kJ} / \mathrm{mol})$ and - $223(\mathrm{~kJ} / \mathrm{mol})$ (Fig. 8b) at 150ns, respectively. Hence the complex of MAPK14 and ligand was dominated by Van der Waals force.

\section{Discussion}

CFF-1 is a classic compound of Chinese traditional medicine which has been used in the clinic to treat patients with prostate cancer in Jiangsu Hospital of Traditional Chinese Medicine for several years as a hospital prescription. Studies have shown that CFF-1 can inhibit the growth of prostate cancer and induce autophagy and apoptosis of prostate cancer cells through multiple signal pathways $[17,18]$. Although some molecular mechanism of CFF-1 inhibiting prostate cancer cells has been studied, the special therapeutic effects of CFF-1 have not been researched. 
In the merge network, the drug active ingredients of licorice could regulate the expression of multiple genes. In addition to licorice, the drug active ingredients of astragalus and Polygonatum could regulate the expression of ESR1 and MAPK14 in the merge network. It has been reported that licorice, astragalus, and Polygonatum can inhibit tumorigenesis in prostate cancer [30-33]. ESR1 is significantly up-regulated in androgen-deprived prostate cancer and promotes tumor cell proliferation [34]. MAPK14 promotes the development and metastasis of cancer $[8,13]$, and is associated with an aggressive risk of prostate cancer [35]. Therefore, CFF-1 inhibits the growth of prostate cancer cells, which might be achieved by inhibiting ESR1 and MAPK14 through licorice, astragalus, and Polygonatum.

In the results of enrichment analysis, some cancer-related signaling pathways were obtained such as Prostate cancer, Pancreatic cancer, Non-small cell lung cancer, colorectal cancer, and so on. The mapped results of the KEGG database show that the number of genes in the cancer signaling pathway is the largest. In the cancer signaling pathway, ESR1 [7] and MAPK14 [13] can promote tumor cell proliferation through the estrogen signaling pathway and MAPK signaling pathway, respectively. AKT1 promotes cancer cell invasion [36] and avoids apoptosis [37] through the PI3K-Akt signaling pathway. CCND1 can be regulated by estrogens to promote the development of prostate cancer cells [38]. Although most of these core genes were concentrated in the cancer signaling pathway, many virus-related signaling pathways also appeared in the KEGG enrichment analysis. Since the same gene plays different functions in different signaling pathways, the virus-related signal pathways were enriched. It is possible that the emergence of virus-related pathways will improve the immunity of patients. The negative correlation between the stemness of tumor cells and immunity indicates that immunity can inhibit the occurrence, development, and metastasis of tumor cells [39]. Therefore, the emergence of virus-related pathways might improve patient immunity and suppress tumors.

In summary, this study employed network pharmacological methods and techniques to identify 112 drug components of CFF-1 and 206 potential targets in prostate cancer. According to the merge network constructed by the core PPI network and the network of active ingredients with target genes, it was found that ESR1 and MAPK14 could be regulated by licorice, astragalus, and Polygonatum. By the enrichment analysis of GO and KEGG, the cancer-related signaling pathway, IL-17, and other signaling pathways were significantly enriched. It could be inferred that CFF-1 might inhibit prostate cancer and promote immune response. And the tool (Do_virtual_screening) we developed could not only realize the virtual screening of drug ingredients but also realize the reverse docking of active ingredients, which provided convenience for future research on TCM. Due to the limitation of the database and software simulation, these results still need to be confirmed by experiments, and the internal implementation mechanism also needs to be explained by related experimental results.

\section{Conclusion}

This study investigated the active ingredients and molecular mechanisms in the prostate cancer treatment of CFF- 1 from the perspective of network pharmacology. The active ingredients of CFF-1 are composed of 112 compounds. There are 206 target genes involved in the treatment of prostate cancer by 
CFF-1, among which ESR1 and MAPK14 are the key target genes. From 112 compounds, the active ingredients that best bind to ESR1 and MAPK14 were obtained with Do_virtual_screening developed by us. Finally, the dynamic process of the binding of two small drug molecules to the target protein was simulated by molecular dynamics. In CFF-1 treated prostate cancer, cancer-related signaling pathways are significantly enriched. Also, the results of this study provide a new way to further research the mechanism of CFF-1 in treating prostate cancer.

\section{Declarations}

\section{Data Availability}

All data generated or analyzed during this study are available.

\section{Conflicts of Interest}

The authors declare no conflict of interest.

\section{Authors' contributions}

Data analysis, writing original draft by Dai Zao; Review and revised the manuscript by Liu Ping. All authors read and approved the manuscript.

\section{Funding Statement}

This research was funded by the National Natural Science Foundation of China (NNSFC) (grant no. 81872104 and 81472415$)$. The funders had no role in research design, data collection, and analysis, decision to publish, or preparation of the manuscript.

\section{Acknowledgments}

Thanks to Dr. Wei Guanyun for her suggestions on data analysis.

\section{Supplementary Information}

Figure S1:Obtained of 206 target genes (a) Target genes related to prostate cancer in five databases; (b) 206 target genes regulated by CFF-1. Table S1: active ingredients of CFF-1 and target genes. Table S2: Intersection genes. Table S3: Molecular docking results of 46 active ingredients of drugs with ESR1 and MAPK14.

\section{References}

1. Siegel RL, Jemal KDMillerA. Cancer statistics 2020 CA: A Cancer Journal for Clinicians. 2020;70(1):7-30. 
2. Nader R. J El AmmJB Aragon-Ching. Role of chemotherapy in prostate cancer. Asian J Androl. 2018;20(3): 221-9.

3. Podder TK, ET FredmanRJ Ellis. Advances in Radiotherapy for Prostate Cancer Treatment. Springer International Publishing, 2018, 1126: 31-47.

4. DE Spratt. Combination therapies in prostate cancer: proceed with caution. Lancet Oncol. 2019;20(3):321-3.

5. Seruga B. A OcanalF Tannock. Drug resistance in metastatic castration-resistant prostate cancer. Nat Rev Clin Oncol. 2011;8(1):12-23.

6. PE ClarkFM Torti. Prostate cancer and bone metastases: medical treatment. Clin Orthop Relat Res. 2003;415(415 Suppl):148-57.

7. Holst F, Stahl PR, Ruiz C, et al. Estrogen receptor alpha (ESR1) gene amplification is frequent in breast cancer. Nat Genet. 2007;39(5):655-60.

8. E HedrickS Safe. Transforming Growth Factor beta/NR4A1-Inducible Breast Cancer Cell Migration and Epithelial-to-Mesenchymal Transition Is p38alpha (Mitogen-Activated Protein Kinase 14) Dependent. Mol Cell Biol. 2017;37(18): MCB.00306-00317.

9. TA StevensR Meech. BARX2 and estrogen receptor-alpha (ESR1) coordinately regulate the production of alternatively spliced ESR1 isoforms and control breast cancer cell growth and invasion. Oncogene. 2006;25(39):5426-35.

10. Ricote M, Garcia-Tunon I, Bethencourt F, et al. The p38 transduction pathway in prostatic neoplasia. J Pathol. 2006;208(3):401-7.

11. Grossi V, Peserico A, Tezil T, et al. p38alpha MAPK pathway: a key factor in colorectal cancer therapy and chemoresistance. World J Gastroenterol. 2014;20(29):9744-58.

12. Bonkhoff $\mathrm{H}$. Estrogen receptor signaling in prostate cancer: Implications for carcinogenesis and tumor progression. Prostate. 2018;78(1):2-10.

13. JY FangBC Richardson. The MAPK signaling pathways and colorectal cancer. Lancet Oncol. 2005;6(5):322-7.

14. Wang Y, Zhang Q, Chen Y, et al. Antitumor effects of immunity-enhancing traditional Chinese medicine. Biomed Pharmacother. 2020;121:109570.

15. Fu B, Wang N, Tan HY, et al. Multi-Component Herbal Products in the Prevention and Treatment of Chemotherapy-Associated Toxicity and Side Effects: A Review on Experimental and Clinical Evidences. Front Pharmacol. 2018;9:1394.

16. L Jun. Clinical Research on 58 Cases of Advanced Non-small Cell Lung Cancer Treated by the Intergrated TCM and Western Medicine [J]. Henan Traditional Chinese Medicine. 2008;6:022.

17. Wu Z, Zhu Q, Yin Y, et al. Traditional Chinese Medicine CFF-1 induced cell growth inhibition, autophagy, and apoptosis via inhibiting EGFR-related pathways in prostate cancer. Cancer Med. 2018;7(4):1546-59. 
18. Zhang Y, Wu ZM, Lei BH, et al. Chinese medicinal compound CFF-1 induces the apoptosis and cyclearrest of prostate cancer cells via the PI3K/AKT/FOXO1 signaling pathway. Zhonghua Nan Ke Xue. 2017;23(9):828-37.

19. Ni MW, Liu XK, Meng ZQ, et al. A bioinformatics investigation into the pharmacological mechanisms of javanica oil emulsion injection in non-small cell lung cancer based on network pharmacology methodologies. Bmc Complementary Medicine Therapies. 2020;20(1):1-18.

20. Zhang LG, Shi XQ, Huang ZQ, et al. Network Pharmacology Approach to Uncover the Mechanism Governing the Effect of Radix Achyranthis Bidentatae on Osteoarthritis. Bmc Complementary Medicine Therapies. 2020;20(1):121.

21. Ru J, Li P, Wang J, et al. TCMSP: a database of systems pharmacology for drug discovery from herbal medicines. J Cheminform. 2014;6(1):13.

22. Yu G, Wang LG, Han Y, et al. clusterProfiler: an R package for comparing biological themes among gene clusters. OMICS. 2012;16(5):284-7.

23. Szklarczyk D, Gable AL, Lyon D, et al. STRING v11: protein-protein association networks with increased coverage, supporting functional discovery in genome-wide experimental datasets. Nucleic Acids Res. 2019;47(D1):D607-13.

24. Shannon P, Markiel A, Ozier O, et al. Cytoscape: A Software Environment for Integrated Models of Biomolecular Interaction Networks. Genome Res. 2003;13(11):2498-504.

25. Tang Y, Li M, Wang J, et al. CytoNCA: A cytoscape plugin for centrality analysis and evaluation of protein interaction networks. Biosystems. 2015;127:67-72.

26. Morris GM, Huey R, Lindstrom W, et al. AutoDock4 and AutoDockTools4: Automated Docking with Selective Receptor Flexibility. J Comput Chem. 2009;30(16):2785-91.

27. 0 TrottAJ Olson. AutoDock Vina: improving the speed and accuracy of docking with a new scoring function, efficient optimization, and multithreading. J Comput Chem. 2010;31(2):455-61.

28. Wallace AC, LaskowskiJM Thornton RA. LIGPLOT: a program to generate schematic diagrams of protein-ligand interactions. Protein Eng. 1995;8(2):127-34.

29. Kutzner C, Pall S, Fechner M, et al. More bang for your buck: Improved use of GPU nodes for GROMACS 2018. J Comput Chem. 2019;40(27):2418-31.

30. Guo SQ, Ma BJ, Jiang XK, et al. Astragalus Polysaccharides Inhibits Tumorigenesis and Lipid Metabolism Through miR-138-5p/SIRT1/SREBP1 Pathway in Prostate Cancer. Front Pharmacol. 2020;11:598.

31. Han S-Y, Hu M-H, Qi G-Y, et al. Polysaccharide from Polygonatum Inhibits the Proliferation of Prostate Cancer-Associated Fibroblasts Cells. Asian Pac J Cancer Prev. 2016;17(8):3829-33.

32. Long T, Liu Z, Shang J, et al. Polygonatum sibiricum polysaccharides play anti-cancer effect through TLR4-MAPK/NF-kappaB signaling pathways. Int J Biol Macromol. 2018;111:813-21.

33. Gioti K, Papachristodoulou A, Benaki D, et al. Glycyrrhiza glabra-Enhanced Extract and Adriamycin Antiproliferative Effect on PC-3 Prostate Cancer Cells. Nutr Cancer. 2020;72(2):320-32. 
34. Shaw GL, Whitaker H, Corcoran M, et al. The Early Effects of Rapid Androgen Deprivation on Human Prostate Cancer. Eur Urol. 2016;70(2):214-8.

35. Zhang Z, Jiang D, Wang $C$, et al. Polymorphisms in oxidative stress pathway genes and prostate cancer risk. Cancer Causes Control. 2019;30(12):1365-75.

36. Shukla S, Maclennan GT, Hartman DJ, et al. Activation of PI3K-Akt signaling pathway promotes prostate cancer cell invasion. Int J Cancer. 2007;121(7):1424-32.

37. Martini M, De Santis MC, Braccini L, et al. PI3K/AKT signaling pathway and cancer: an updated review. Ann Med. 2014;46(6):372-83.

38. Nakamura Y, Felizola SJ, Kurotaki Y, et al. Cyclin D1 (CCND1) expression is involved in estrogen receptor beta (ERbeta) in human prostate cancer. Prostate. 2013;73(6):590-5.

39. Miranda A, Hamilton PT, Zhang AW, et al. Cancer stemness, intratumoral heterogeneity, and immune response across cancers. Proceedings of the National Academy of Sciences. 2019;116(18): 9020.

\section{Figures}




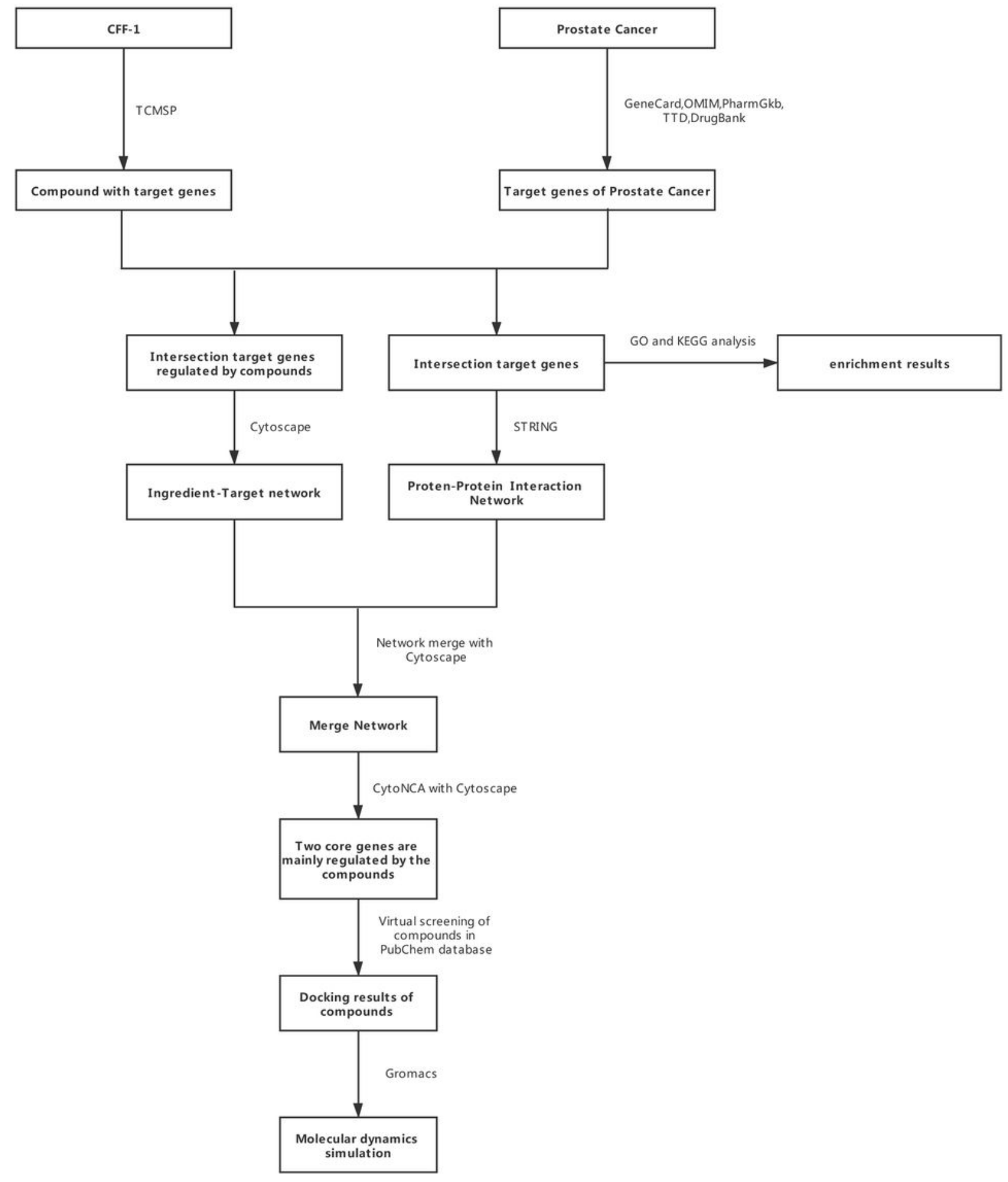

Figure 1

whole flowchart based on network pharmacology 

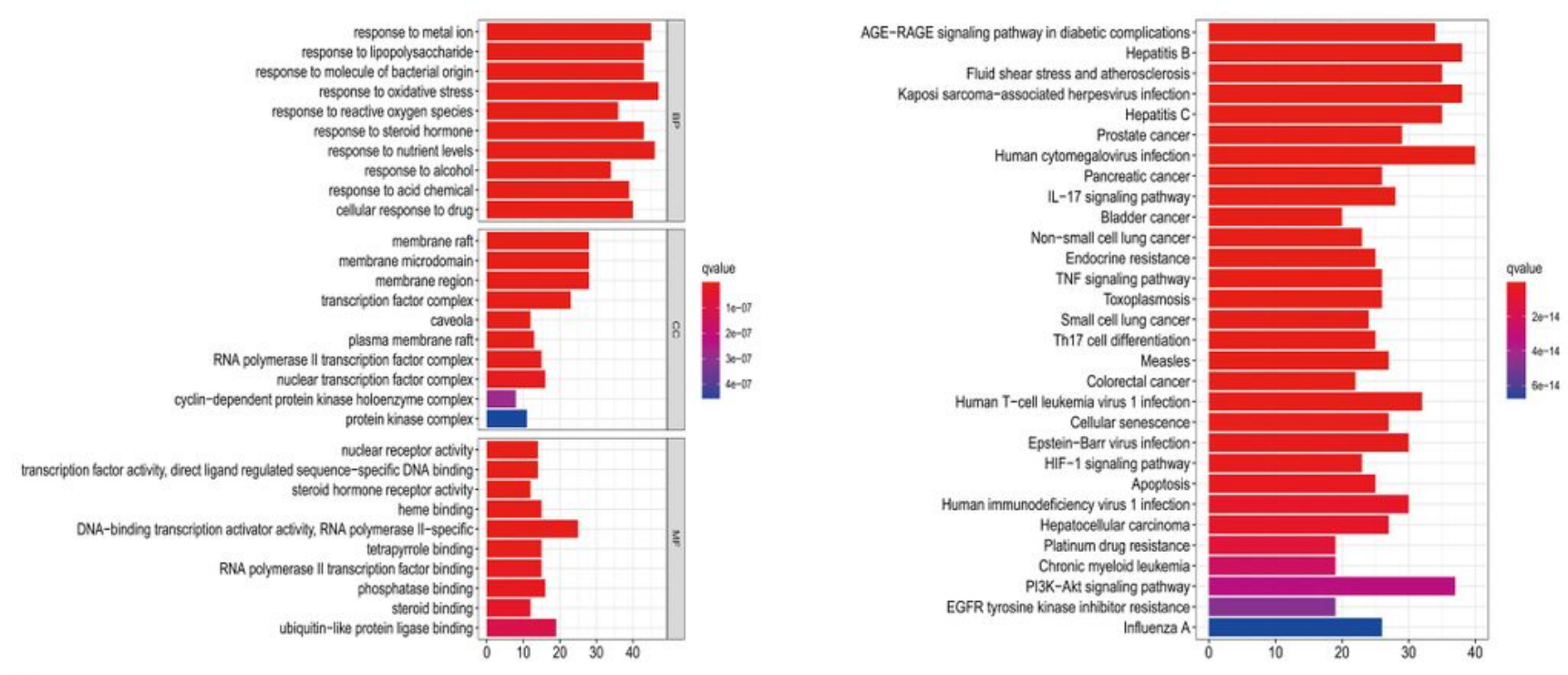

$\mathrm{c}$

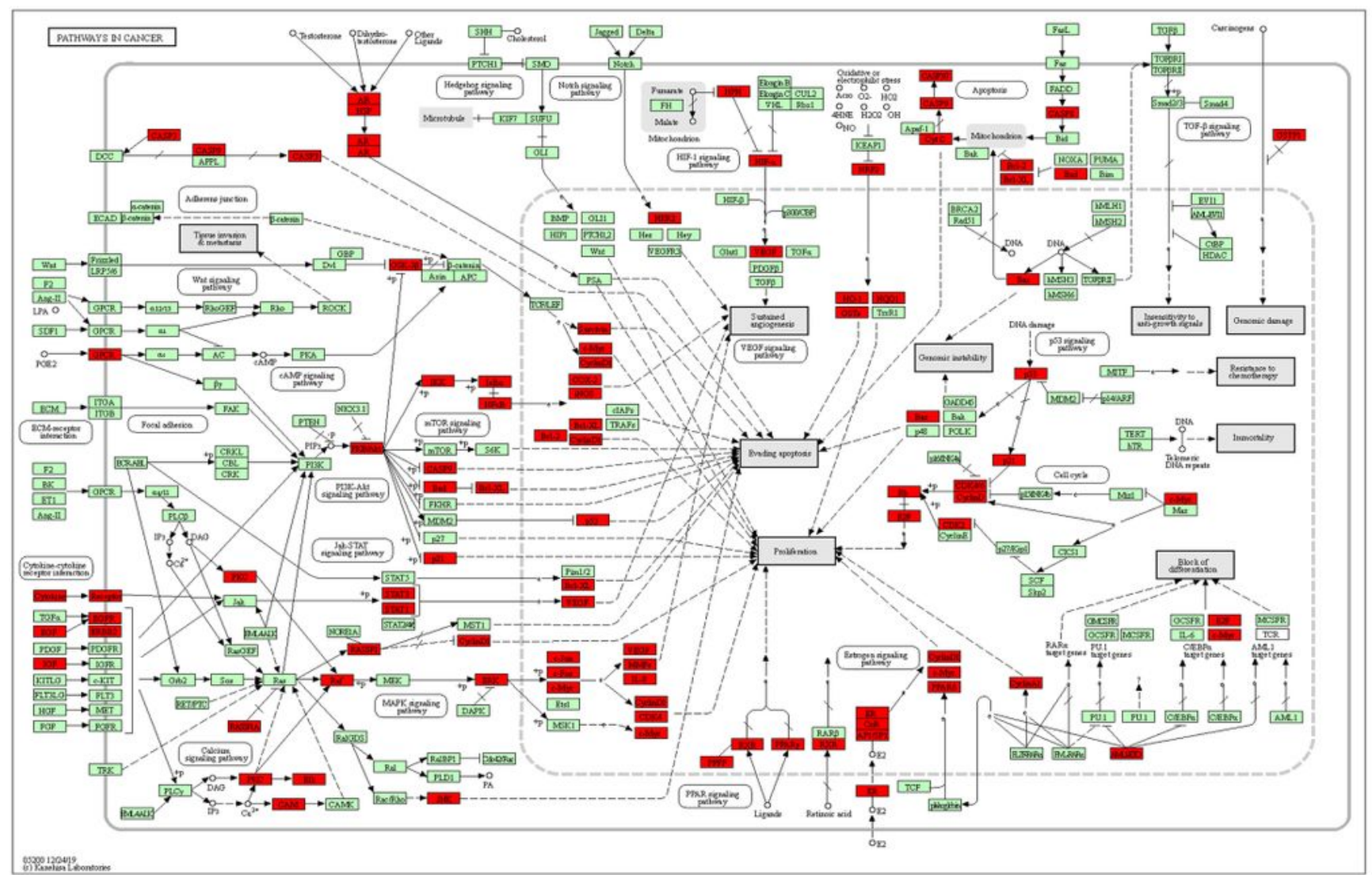

Figure 2

The results of GO and KEGG enrichment analysis (a) The results of GO enrichment analysis; (b) The results of KEGG enrichment analysis; (c) Pathway map of CFF-1 in the treatment of prostate cancer. Red represents the target genes suppressed by the active ingredient of CFF-1 


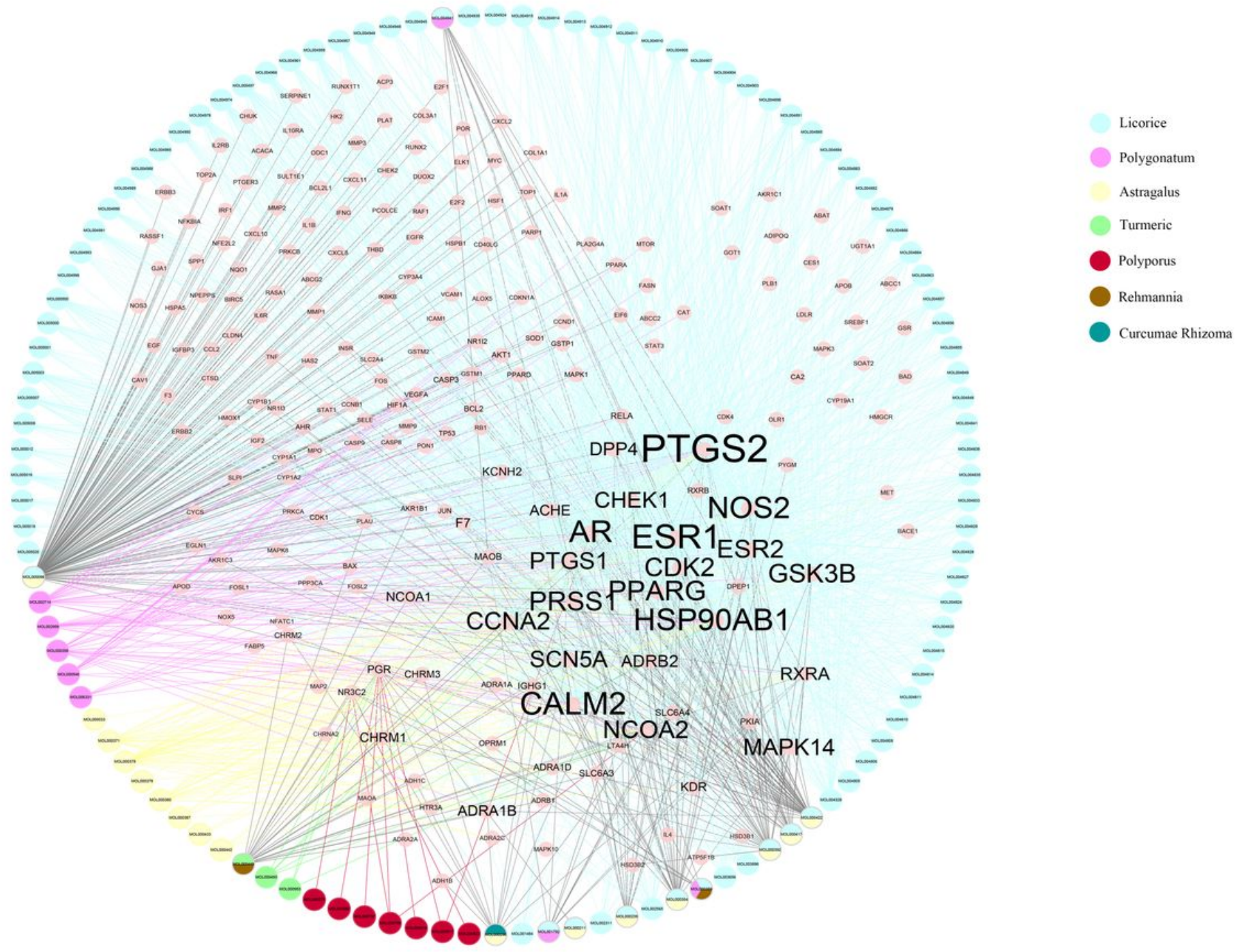

Figure 3

The network of CFF-1 active ingredients and target genes Different colors represent different Chinese medicine ingredients. The grey lines represent ingredients from multiple Chinese medicine ingredients. 
a

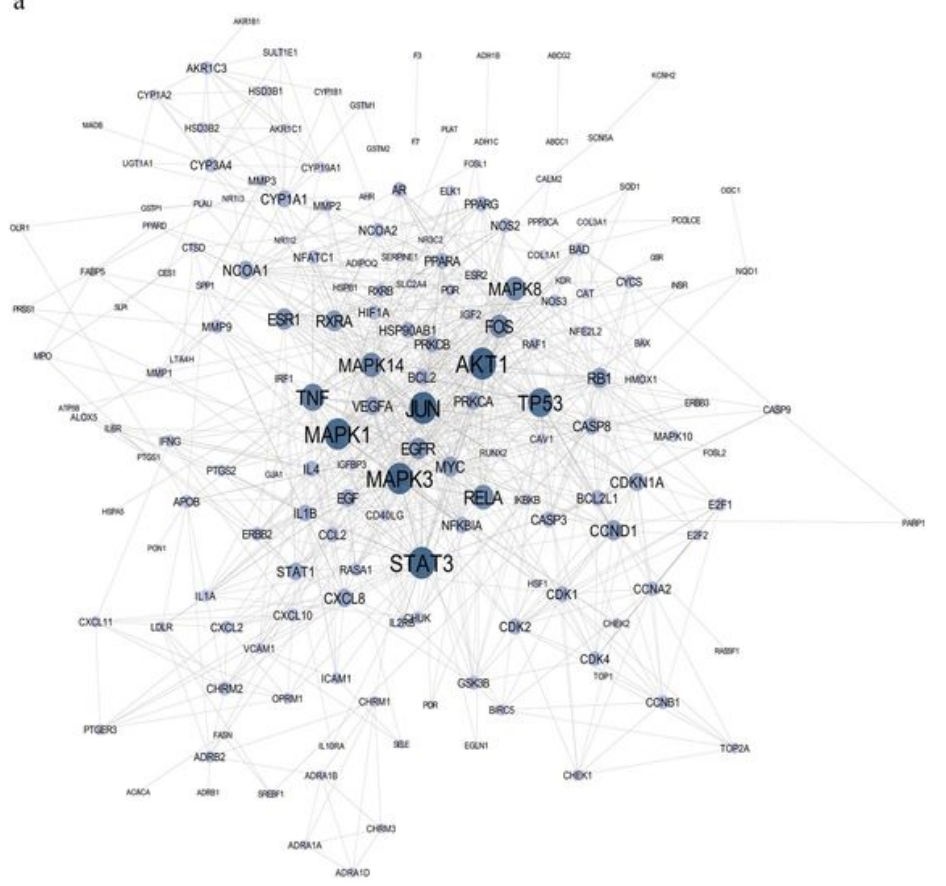

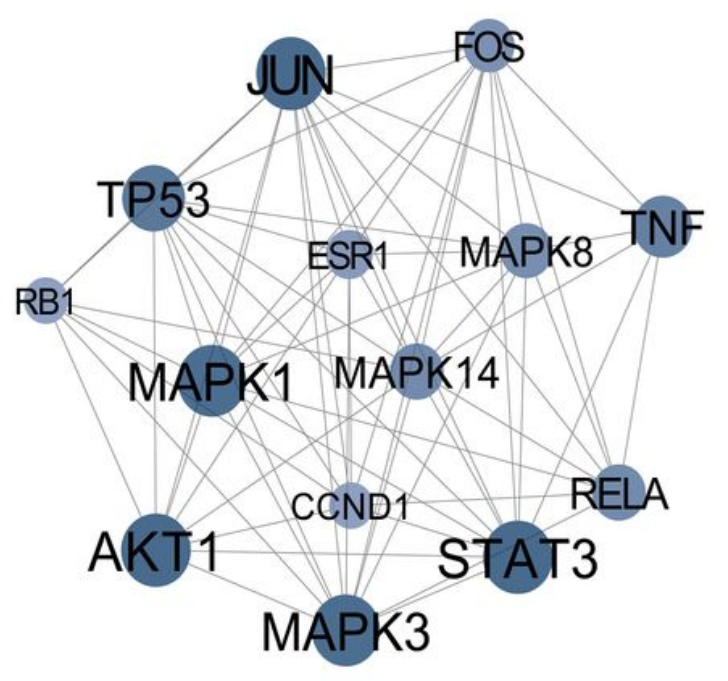

Figure 4

The network of protein-protein interaction network (a) Protein-protein interaction network of 206 target genes; (b) Protein-protein interaction network of core genes. 


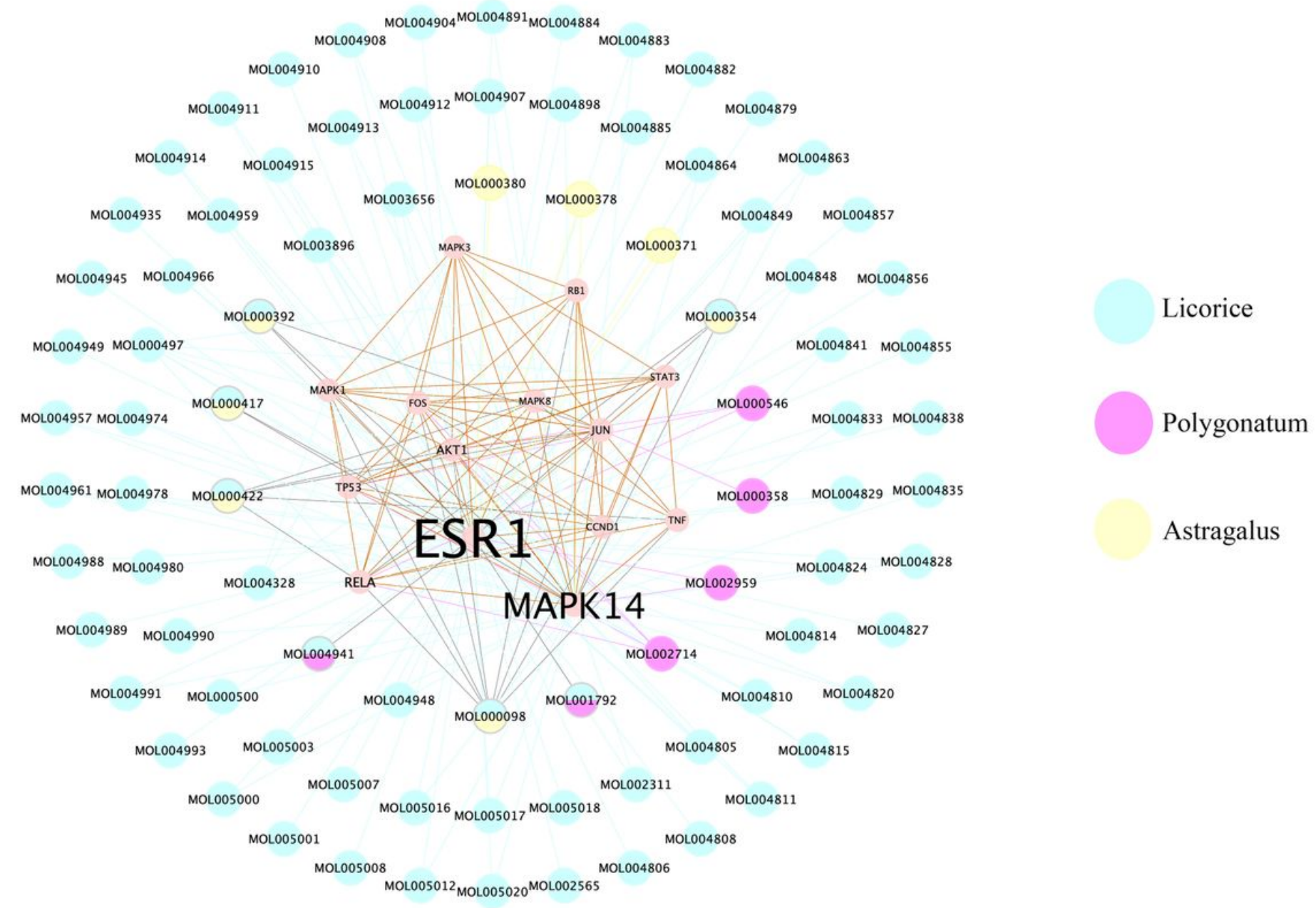

Figure 5

A merge network of CFF-1 active ingredient network and core protein-protein interaction 
a
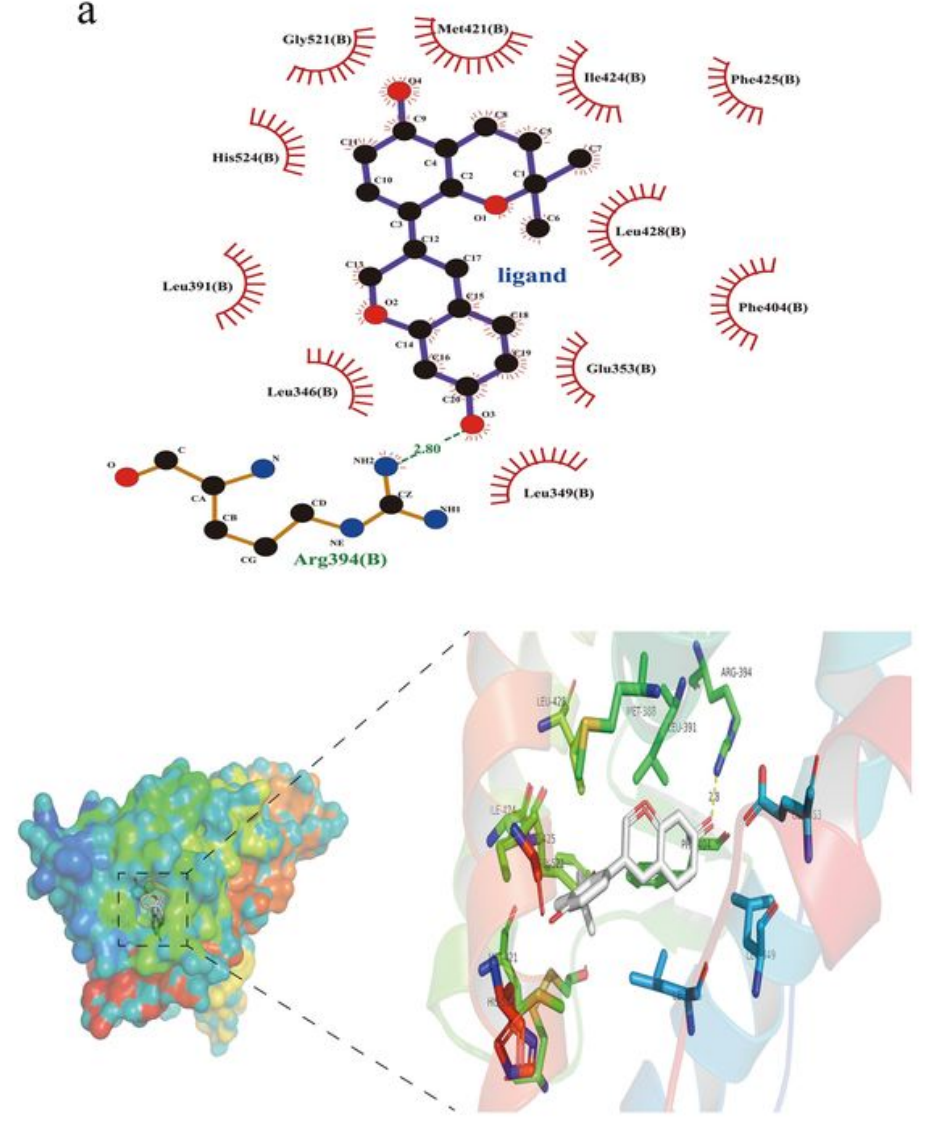

b
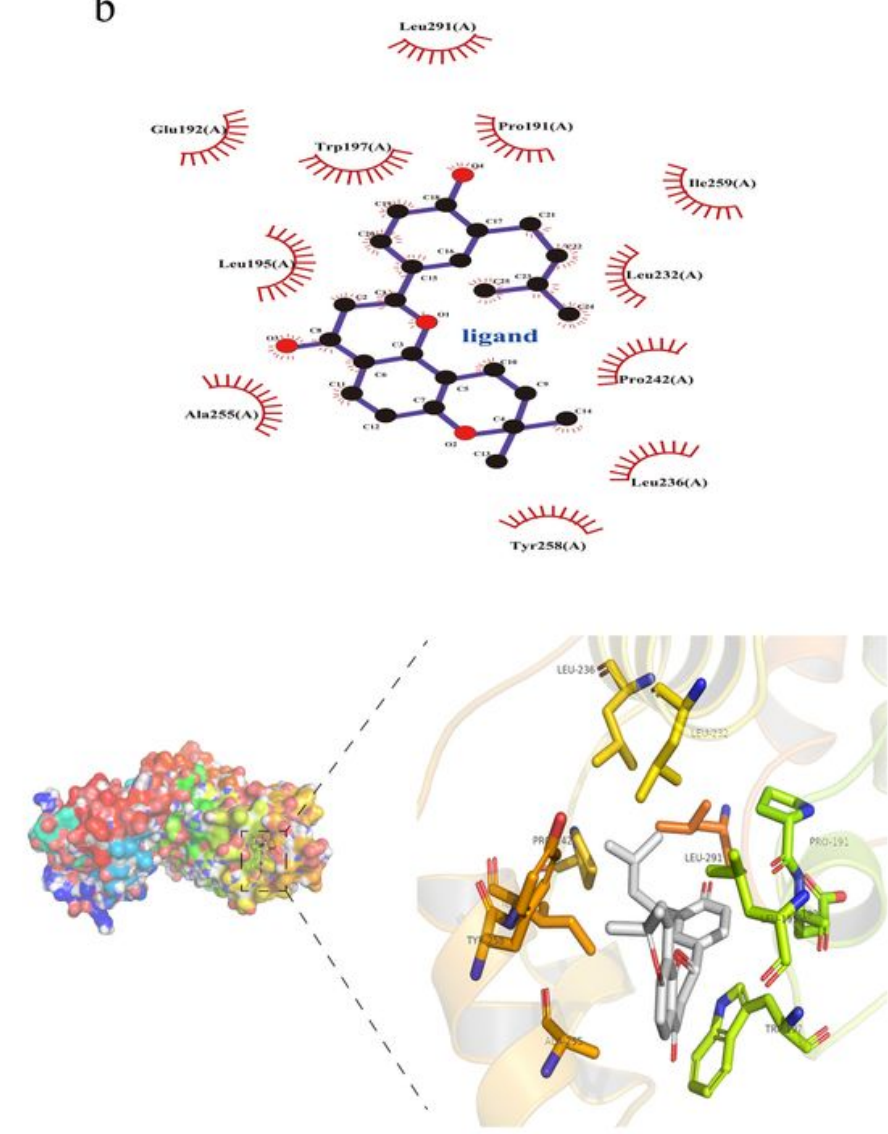

Figure 6

Results of molecular docking between ESR1 and MAPK14 (a) 2D and 3D images of ESR1, including a 2.8nm hydrogen bond and multiple hydrophobic interactions; (b) 2D and 3D images of MAPK14, including multiple hydrophobic interactions.

a

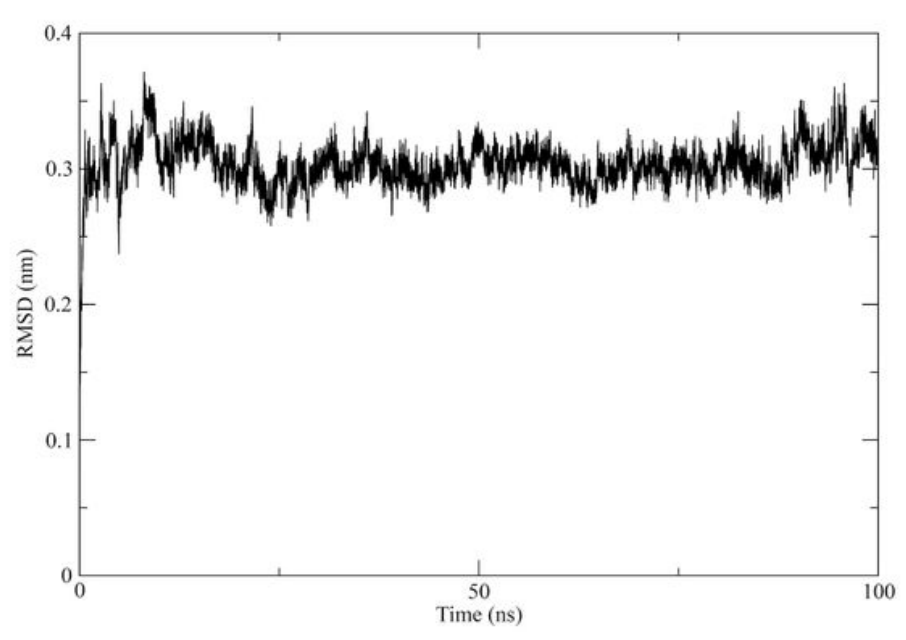

b

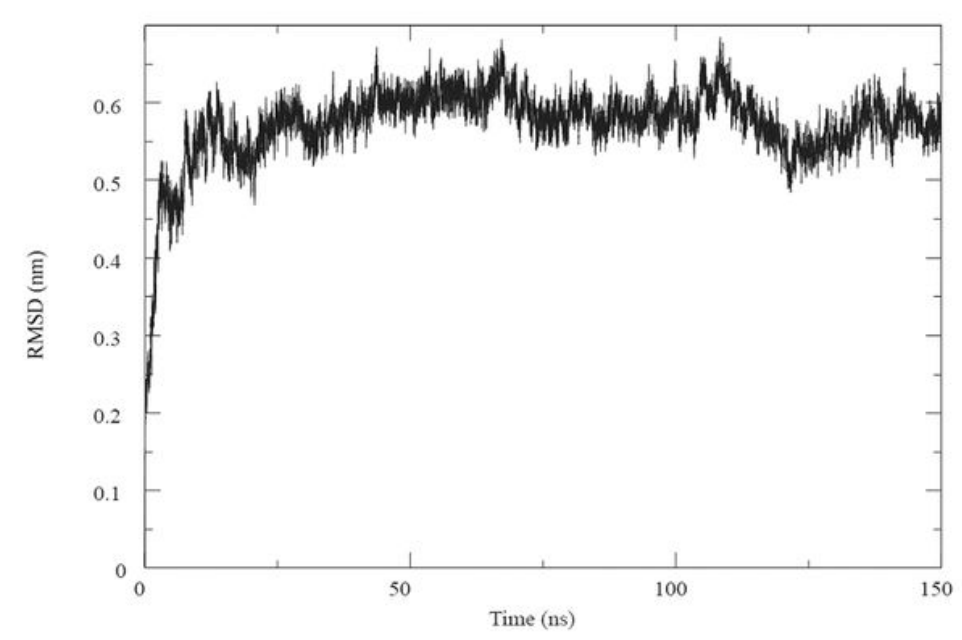

Figure 7 
The RMSD value of protein and ligand (a) RMSD curve of ESR1 and ligand; (b) RMSD curve of MAPK14 and ligand.

a

\section{GROMACS Energies}

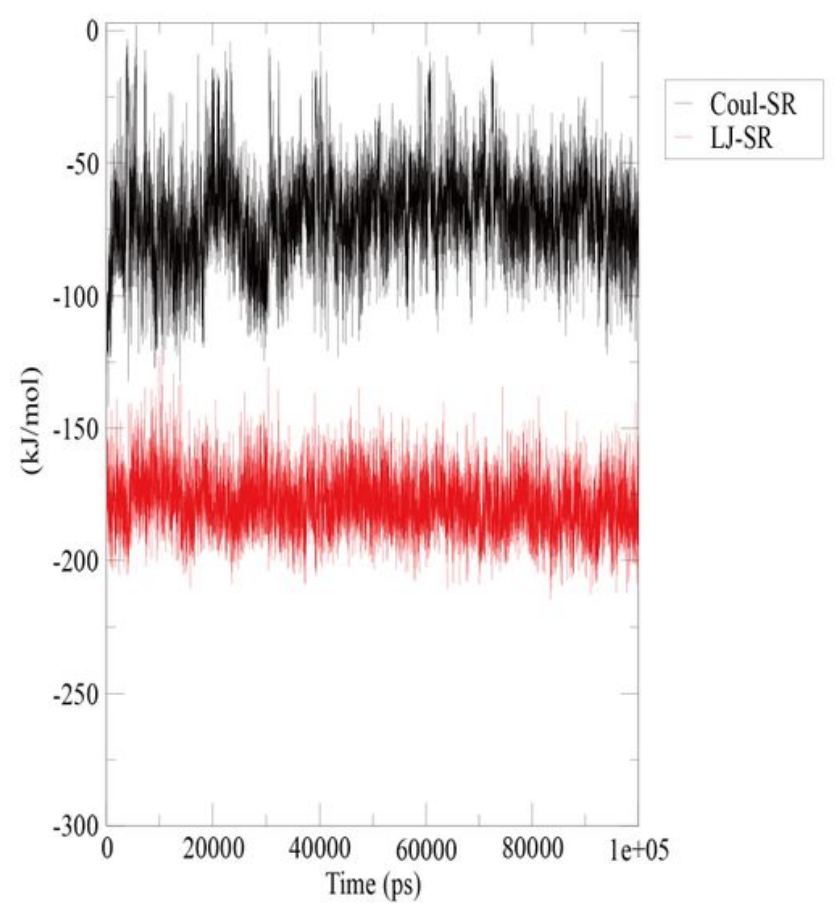

$\mathrm{b}$ GROMACS Energies

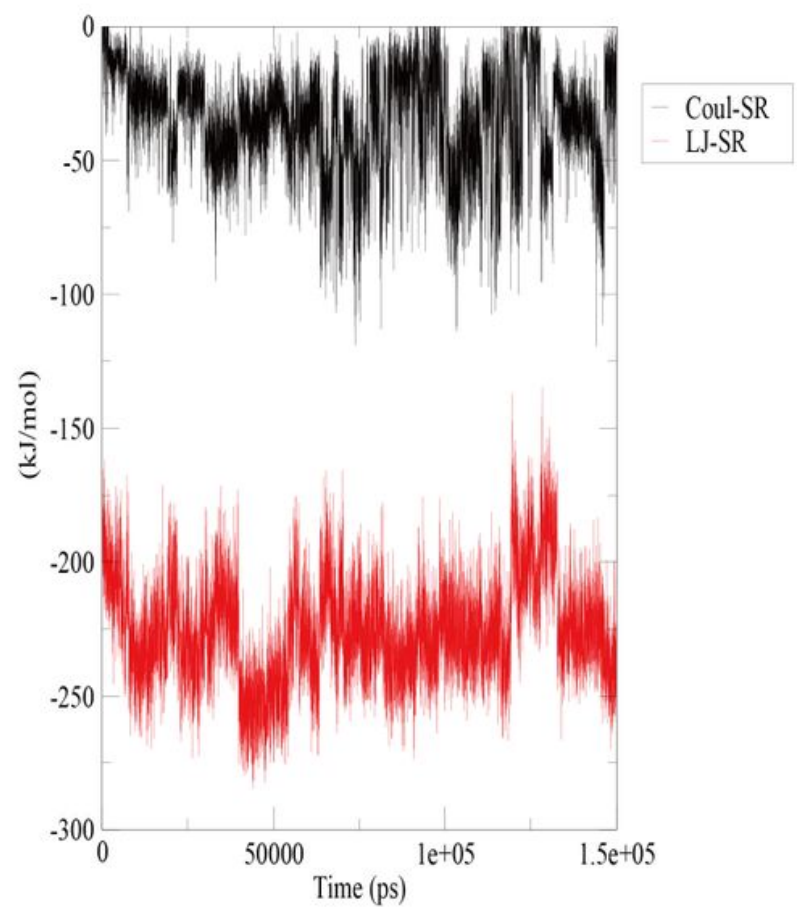

Figure 8

Coulomb and Van der Waals force of protein and ligand (a) Coulomb force and Van der Waals force of ESR1 and ligand; (b) Coulomb force and Van der Waals force of MAPK14 and ligand.

\section{Supplementary Files}

This is a list of supplementary files associated with this preprint. Click to download.

- FigureS1.jpg

- Tables1.xls

- Tables2.xls

- Tables3.xls 